\title{
Intensified Doxorubicin-Based Regimen Efficacy in Residual Non-Hodgkin's Lymphoma Disease: Towards a Computationally Supported Treatment Improvement
}

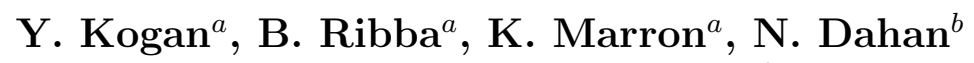 \\ V. Vainstein ${ }^{a}$, and Z. Agur ${ }^{a, b 1}$ \\ ${ }^{a}$ Institute for Medical Biomathematics (IMBM), POB 282 \\ Hate'ena 10, Bene Ataroth, 60991 Israel \\ ${ }^{b}$ Optimata Ltd, 7 Abba Hillel Silver St., Ramat-Gan, 52522 Israel
}

\begin{abstract}
Despite recent advances, treatment of patients with aggressive Non-Hodgkin's lymphoma $\left(\mathrm{NHL}^{2}\right)$ has yet to be optimally designed. Notwithstanding the contribution of molecular treatments, intensification of chemotherapeutic regimens may still be beneficial. Hoping to aid in the design of intensified chemotherapy, we put forward a mathematical and computational model that analyses the effect of Doxorubicin on NHL over a wide range of patho-physiological conditions. The model represents tumour growth both in diffusion-limited settings, that is, in small avascular tumours and tumour cords, and in perfusion-limited settings, e.g. in well-vascularized tumours. Model simulations indicated the presence of a critical regimen intensity below which treatment will fall short of tumour elimination. Taking this critical intensity into account, we compared two regimen intensification strategies: Dose escalation and regimen densification, i.e. reducing the inter-dosing interval. In the diffusion-limited setting, dose escalation was somewhat more efficient than regimen densification. In the perfusion-limited setting, both intensification strategies yielded similar results. The present study coupled with a realistic myelotoxicity model may add insight on the optimisation of NHL intensified chemotherapy design.
\end{abstract}

Key words: mathematical model, vascular tumour, avascular tumour, heterogeneity, chemotherapy, Non-Hodgkin Lymphoma, CHOP, Doxorubicin, hybrid cellular automata

\footnotetext{
${ }^{1}$ Corresponding author. E-mail: agur@imbm.org

${ }^{2}$ Non-standard abbreviations: (i) CI - Critical Intensity; (ii) NHL - Non-Hodgkin's Lymphoma
} 
AMS subject classification: 92C50, 37N25, 37B15, 35Q80, 37M05, 92B05

\section{Introduction}

Non-Hodgkin's lymphomas (NHL) are neoplastic transformations of lymphoid tissue cells [10]. According to the statistics of USA Leukemia \& Lymphoma Society about 58,870 new cases of NHL were expected to be diagnosed in the United States in 2006 alone, where the disease ranks as the sixth leading cause of cancer-related death [13]. Treatment of NHL poses a difficult clinical problem, and at present less than $35 \%$ of all diagnosed cases are cured. The majority of patients are treated with the CHOP [12] chemotherapy regimen, consisting of 4-8 treatment cycles administered at 21-day intervals. Despite several decades of clinical investigation, CHOP remains the first line treatment in NHL [8]. Several questions concerning NHL chemotherapy remain as yet unresolved. Among these are the number of dosing cycles required for maximum treatment efficacy, the optimal dose to be applied at each cycle, and the interval between drug applications. Chemotherapeutic regimen intensity, defined as drug dose divided by dosing interval is a combined parameter used in medicine for comparing different schedules. Recent clinical trials of intensified CHOP regimens (dose application every 14 instead of 21 days) given to NHL patients have produced promising results $[12,40]$; see also $[18,23,26]$. While addition of anti-B-cell monoclonal antibodies (Rituximab) to the regular CHOP regimen yields clinical benefit in NHL treatment [20], clinical trials evaluating the combination of Rituximab with intensified CHOP are underway.

The optimal approach to intensified chemotherapy regimen design has not yet been determined. The possibilities include increasing the cytotoxic drug dose administered per cycle (denoted dose escalation), reducing the dosing interval between the cycles (denoted regimen densification), or some combination of the two. Each approach has its own advantages: while dose escalation can affect more cells per drug application, regimen densification allows less time for tumour recovery between consecutive treatment cycles and less time for chemoresistance to appear. Therefore, the superiority of one strategy over the other is not intuitively evident. In one small clinical trial of intensified CHOP in NHL patients, both dose escalation and regimen densification led to similar efficacy [28].

In order to evaluate the different approaches to regimen intensification a large number of potential treatments has to be tested and it seems impossible to evaluate all of them by the usual experimental method of trial-and-error. A more feasible approach is to mathematically model disease dynamics in conjunction with the relevant drug pharmacokinetics (PK) and pharmacodynamics (PD) and to carry out exhaustive theoretical analysis of the trade-off between the two regimen intensification strategies by simulating many putative regimens in the computer. A simple mathematical model, based on statistical data of patient response, has already been applied in Hodgkin's lymphoma research for performing such a comparison [25]. This study, suggesting that dose escalation 
is more effective than schedule densification, is yet to be confirmed in clinical trials. The above model does not take into consideration the effects of tumour heterogeneity on the patient's overall response. However, in real-life tumour spatial structure and physiology can be highly heterogeneous, some regions of the tumour being well-vascularized, allowing all cells in the area to be sufficiently nourished, whereas other regions may receive a limited blood supply. This factor may be expected to influence the efficacy of different drug treatment regimens. Therefore, it needs to be taken account of in the mathematical model describing vascular tumour growth dynamics under different drug regimens.

In order to allow for tumour heterogeneity when evaluating different methods of regimen intensification, we developed a new mathematical model for drug-disease interactive dynamics, which differs from Hasenclever [25] in having a higher level of detail.

Importantly, our model considers two patho-physiological scenarios: Diffusion-limited and perfusion-limited [29]. The diffusion-limited setting describes tumour regions with relatively low vascular density and high blood flow (e.g. small avascular tumours and tumour cords). In this case, nutrient supply is determined primarily by its ability to diffuse from the blood vessels into inner tumour areas. In the present work the diffusion-limited scenario represents avascular spheroid tumours surrounded by a medium with a high nutrient concentration, and vascular tumours consisting of a network of blood vessels sparsely intermingled with tumour cell clusters. The perfusion-limited scenario describes well-vascularized regions of the tumour in which blood flow (perfusion) constitutes a decisive factor in nutrient supply. However, blood flow is subject to varying degrees of temporal changes $[29,42,41,49]$. Therefore, in the perfusion-limited setting, we model vascular tumours with both high and low degrees of temporal heterogeneity (see Figure 1).

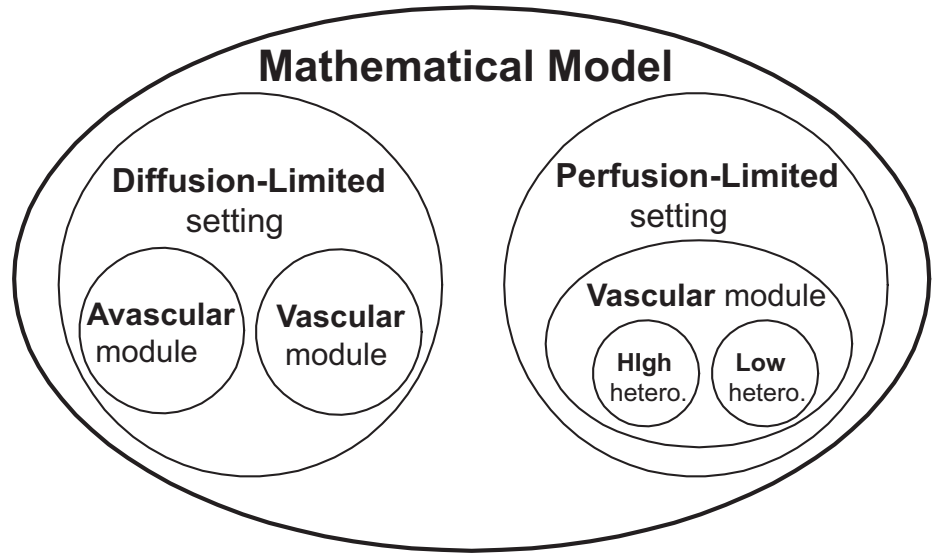

Figure 1: Scheme of the mathematical model. The model considers tumour growth in diffusion-limited and perfusion-limited scenarios. The diffusion-limited scenario can encompass avascular and vascular tumours, whereas the perfusion-limited scenario consists of vascular tumours. In the latter scenario, both high and low levels of temporal blood flow heterogeneity are modelled (denoted High Hetero. and Low Hetero. respectively).

Many avascular tumour models have been studied previously $[15,16,17,35]$. These models 
describe spatial structure, nutrient and drug diffusion and cell kinetic characteristics (proliferation, quiescence, necrosis, etc.). As our work is aimed at describing the effect of a cell-cycle phase specific drug, our avascular tumour growth model is new in incorporating a detailed description of the cell cycle into the traditional avascular tumour growth model. Vascular tumour growth have previously been modelled by many, including Liotta et al. [34], Orme and Chaplain [38], Agur et al. [2], Ribba et al [43], Jana et al., [30] and Arakelyan et al. [7]. The latter model was validated experimentally [6]. The vascular tumour module described here is based on Ribba et al. [43], including the cellular automata (CA) processing rules.

The model brought forward here describes tumour dynamics on the cellular level and tumour geometry corresponding to microscopic residual disease behaviour. The pharmacokinetic (PK) and pharmacodynamic (PD) properties of Doxorubicin that are modelled in conjunction with tumour dynamics, enable to identify conditions under which regimen intensification can improve treatment efficacy of a residual NHL disease.

\section{Methods}

\subsection{Mathematical modelling}

The mathematical model which describes the NHL dynamics under Doxorubicin therapy, incorporating both diffusion-limited and perfusion-limited settings, is described hereafter. We use a different formalism for each of the two settings. Model's equations are described in the appendix.

\subsubsection{Avascular tumour (spheroid) growth module}

2.1.1.1. General. Our model describes avascular tumours in the form of spheroids that consist of stacked spherical layers containing proliferating, quiescent and necrotic NHL cells. It describes the dynamics of cell populations in the successive spherical layers, regulated by the nutrient and drug concentration available through diffusion from the outer micro-environment. As each layer of the structure can only contain a limited number of cells, geometrical rearrangement occurs, meaning that cells can move from one layer to another and new peripheral layers are generated. The main processes modelled in the detailed avascular tumour growth module are: (i) diffusion of nutrient and drug into the tumour through the different inter-cellular and intra-cellular media; (ii) NHL cellular dynamics, including cell proliferation, cell-cycle arrest and necrosis; (iii) geometrical rearrangement of cells within the growing tumour.

2.1.1.2. NHL cell dynamics in the growing spheroid. Cell behaviour is determined by its geometric position and the concentration of nutrients and drug in its micro-environment, which is computed by the diffusion equations. At each moment the proportion of proliferating cells, but not quiescent cells, is eliminated by the drug (see below in this section for details and experimental support). Depending on the local nutrient 
concentration, proliferating cells may go into quiescence, and vice versa. Surviving proliferating cells advance in their cell-cycle phase, dividing into new proliferating cells upon ion of the $\mathrm{G} 2+\mathrm{M}$ phase. A certain proportion of the viable cells enters necrosis according to local nutrient concentration and disappears after a fixed necrosis period. 2.1.1.3. Geometrical rearrangement. At any moment, the total number of cells in the avascular tumour may increase or decrease due to proliferation or death. As a result, the tumour undergoes geometrical rearrangement, so that layers are added to the structure or removed from it. Geometrical rearrangement is onset at the outer layer and directed inwardly, i.e. spare cells move towards the centre of the structure. Consequently, outer layers may be filled, whereas inner layers tend to be sparse [31].

\subsubsection{Vascular tumour growth module}

2.1.2.1. Two-dimensional vascular tumour model. In the present work, we described vascular tumours using a vascular tumour growth model developed by Ribba et al. [43], which is based on the hybrid CA, previously applied to different aspects of tumour growth $[4,21,39]$. The model's two-dimensional framework consists of a $2 \mathrm{~mm}$ square tissue composed of 110 blood vessels. The vessels make up a simple vascular network, corresponding to a structure and density that have been observed in tissues such as the liver and colon $[32,48]$. The vessels' radii are subject to modification through various vessel structural modification processes $[42,41,49]$. The domain is initially filled with NHL cells forming one random pattern or more.

The vascular structure takes into account blood flow heterogeneity, which is a significant factor in cancer growth and treatment [29]. It describes the development of individual cells, which obtain nutrients and drug through diffusion from the blood vessels. We describe here the main processes simulated by the vascular module, namely: (i) blood flow and vessel structural modification; (ii) nutrient and drug diffusion; (iii) cell dynamics.

2.1.2.2. Blood flow and vessel structural modification. Our model assumes the blood flow in each vessel to be laminar steady Poiseuille flow (see Appendix). The vasculature is subject to remodelling, which occurs according to two different mechanisms. The first is the so-called structural adaptation mechanism, which describes the contraction and expansion of mature blood vessels, enabled by smooth muscle cells and pericytes that encase the vessel walls. Structural adaptation occurs according to flow, in particular wall shear stress $[42,41]$. The second remodelling mechanism is vessel maturation and the destabilization of mature vessels. In cancer, due to neovascularization, a large proportion of the vessels is immature [11]. Unlike mature vessels, immature vessels are not surrounded by pericytes and smooth muscle cells. Therefore, following Arakelyan et al. [7], immature vessels are included in our model but are considered incapable of structural adaptation. As a result, their configuration is less stable: Their radii are subject to random change and tend to be larger than the radius of mature vessels, increasing leakage. In order to incorporate these characteristics, the immature vessels' radii are randomly modified at each simulation step (see Appendix). 
As the NHL cell cluster grows, it engulfs nearby blood vessels (a process denoted cooption). Our model considers that once a vessel is surrounded by NHL cells, its pericyte layer destabilizes. The status of a vessel (mature or immature) is continually updated, allowing immature vessels to become mature once again, as occurs in actual vascular tumours [49]. We also defined the vascular network's sensitivity to cooption as negatively related to the number of surrounding cells required to destabilize a blood vessel. When the network is highly sensitive to cooption, blood flow is destabilized, leading to temporally heterogeneous nutrient distribution and to inconsistent tumour growth. In the case of low sensitivity the blood flow is not as easily perturbed, therefore tumour growth is more stable.

2.1.2.3 Cell dynamics. To accommodate the above-mentioned vascular properties, cell dynamics in the vascular module are implemented as CA rules, as opposed to the avascular module in which the population is modelled as a whole. The cell dynamics are modelled in the vascular module similarly to those in the avascular, with the following modifications:

(i) the vascular module considers proliferating and quiescent cells, but not necrotic process: once a cell dies it disappears immediately from the system; (ii) transition from proliferation to quiescence and vice versa, in addition to being regulated by the local nutrient concentration, depends also on over-crowdedness [5]; (iii) when a cell divides, daughter cells move towards higher nutrient concentrations.

\subsubsection{Doxorubicin PK/PD}

We described Doxorubicin PK using the so-called one-compartment model, which reflects the decline of drug concentration in plasma over time. Doxorubicin is known to act on both proliferating and quiescent cells. However, the effect on quiescent cells can be assumed negligible [9]. At each moment, the local drug concentration is determined according to diffusion or flow equations. According to this concentration, a proportion of proliferating cells is eliminated and the percentage of cells surviving the drug ("survival fraction") can be calculated (see Appendix for full PK/PD equations).

\subsubsection{Nutrient and drug diffusion}

We assumed that in avascular tumours nutrient and drug concentrations directly regulate cell dynamics, including proliferation and death. In describing the diffusion of these chemicals from the outer environment through the successive cell layers into the tumour core, we used a classical diffusion equation, which assumes spherical geometry and symmetry for initial and boundary conditions. We modified this equation to take into account viable cells' consumption of nutrient and drug. The diffusion equations of the model are presented in Appendix. We further assumed that in well-vascularized tumours the maximum distance between a cell and the blood vessel nearest to it is small $(80 \mu \mathrm{m})$, therefore we applied the adiabatic approximation according to which chemicals diffuse instantaneously. We described the diffusion process using a standard diffusion equation, modified to incorporate the local uptake of nutrient and drug (see Appendix). 


\subsection{Treatment efficacy: Comparison between regimens}

The intensity of a chemotherapy regimen is defined as the amount of drug $(\mathrm{mg} / \mathrm{m} 2)$ per unit of time (day or week) [27]. In this study, we simulated and compared three different Doxorubicin treatment regimens: a standard regimen, consisting of six "standard" doses ${ }^{3}$ administered once every 21 days, and two intensified regimens: (i) a dose-escalated regimen, in which the interval between cycles is maintained at 21 days and the dose per cycle is doubled; (ii) a densified regimen, in which the "standard" dose is maintained and the interval between cycles is shortened to 10 days. The same total dose was applied in all three regimens. Thus, both intensified treatment regimens were twice the intensity of the standard regimen.

\subsubsection{Assessment of regimen efficacy}

In clinical studies, treatment efficacy is usually measured by disease-free survival and overall survival. Any mathematical or computational model is too simplistic to retrieve these global measurements. In our simulations, we evaluated treatment efficacy according to the following three criteria [43]:

2.2.1.1. Residual tumour volume. We defined the residual tumour volume of treated NHL as $\left(n^{\prime} / n\right) \times 100 \%$, where $n$ and $n^{\prime}$ are, respectively, the tumour size (in terms of number of cells) at the beginning and at the end of the simulation.

2.2.1.2. Average tumour regrowth following Doxorubicin application cycle. We calculated the tumour's rate of regrowth following each drug application, i.e. the ratio of tumour size at the end of each drug cycle (denoted $n_{(x+1)}$ ), immediately preceding the next dosing, to its size at the beginning of the cycle, prior to drug application (denoted $n_{(x)}$ ):

$$
R_{(x)}=\frac{n_{(x+1)}}{n_{(x)}} \times 100 \% .
$$

2.2.1.3. Time below threshold. We calculated the percentage of time in which the tumour size was maintained below a certain threshold, defined as a proportion of the tumour's original size. For example, we computed the percentage of time the tumour size remained below $S_{1 / 2}$ (half of its original size) as follows:

$$
P_{1 / 2}=\frac{\sum_{t=0}^{T} H\left(S_{1 / 2}-s(t)\right)}{T} \times 100 \%,
$$

where $P_{1 / 2}$ is the percentage of time for which the tumour is below half its initial size, $T$ is the total duration of the simulation, $s(t)$ is the size of the tumour at time $t$, and $H$ is the Heaviside function:

$$
H(x)=\left\{\begin{array}{l}
1 \text { if } x \geq 0 \\
0 \text { if } x<0
\end{array} .\right.
$$

\footnotetext{
${ }^{3}$ The vascular and avascular modules are each accorded their own "standard" dose; see "Results" section for further details.
} 
The thresholds we examined were $S_{3 / 4}, S_{1 / 2}$ and $S_{1 / 4}$.

\subsection{Parameter Evaluation}

The parameter values used in the above equations were taken from literature $[14,22,29$, $34,37,38,39,41,42,43,44]$. Main model parameters and their values are presented in Table 1. Cell kinetic parameters implemented in the model are presented in Table 2 (applicable to High-Grade NHL ${ }^{4}$ ). The number of cells and the geometrical dimensions of the models used in simulations (order of magnitude of few millimeters) correspond to the residual disease.

\section{Results}

\subsection{Critical regimen intensity}

First, we wished to determine the relationships between regimen intensity and treatment efficacy. To this end, we simulated the model under various treatment schedules, increasing the intensity either by dose escalation or by regimen densification. Simulations indicated that the relationship between regimen intensity and treatment efficacy is not linear.

Rather, there is a critical intensity above which further regimen intensification will lead to significant improvement, but below which any treatment is ineffective: Despite drug application, the tumour is larger at the beginning of each treatment cycle than it was at the beginning of the previous cycle. This critical intensity can also be clearly observed when comparing the effects of different regimen intensities on the percentage of time the tumour is maintained below the $S_{1 / 2}$ threshold (Figure 2). Though Figure 2 displays particular results of regimen intensification through dose escalation in the diffusion-limited setting, the existence of this critical intensity was demonstrated in all modelled pathophysiological scenarios, whether treatment was intensified through dose escalation or through regimen densification (not shown). Thus, the "standard" dose assigned to each modelled scenario was the minimal dose above which further increase had an effect.

\subsection{Comparison between different strategies of treatment intensification}

To compare different strategies of treatment intensification we simulated three Doxorubicin regimens. The standard regimen was compared to dose-escalated and densified regimens (for details see Methods). The analysis was performed separately for the following four model scenarios: (i) avascular (diffusion-limited); (ii) vascular, diffusion-limited; (iii) vascular, perfusion-limited, with high heterogeneity; (iv) vascular, perfusion-limited, with low heterogeneity (see Introduction).

\footnotetext{
${ }^{4}$ NHL cases are characterized by a grade of aggressiveness, which can be low, intermediate or high. In this study we investigated High-Grade NHL.
} 

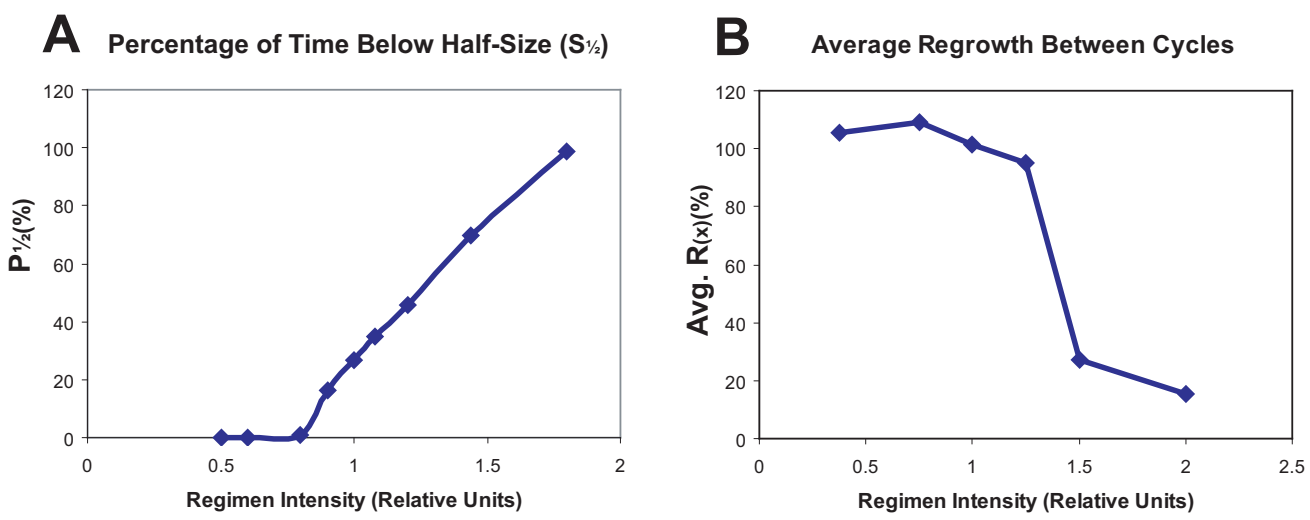

Figure 2: Efficacy of simulated Doxorubicin treatment regimens as a function of regimen intensity (dose/dosing interval). In the results shown, regimen intensity is varied through dose escalation, i.e. varying the administered dose while maintaining the dosing interval at 21 days. Critical regimen intensity must be reached in order to improve treatment efficacy. 2A: Percentage of time the simulated tumour is maintained below half of its original size as a function of regimen intensity. Results apply to avascular tumours in the diffusion-limited scenario. 2B: Average cycle tumour regrowth between drug administrations (tumour size at end of dosing /tumour size at beginning of dosing cycle) as a function of regimen intensity. Results apply to vascular tumours in the diffusion-limited scenario.

Figure 3 depicts simulated tumour dynamics over time under standard, dose-escalated and densified treatment regimens for each of the four model scenarios. Below we describe the results of the comparison according to three criteria of treatment efficacy described in Methods section: residual tumour volume, average tumour regrowth and time below threshold.

\subsubsection{Residual tumour volume}

The average residual tumour volume obtained under each simulated treatment regimen is presented in Table 3. In all scenarios examined, the intensified regimens produced a significantly lower residual tumour volume than the standard regimen. In the diffusion-limited scenario, the dose-escalated regimen was more effective than the densified regimen: in the avascular case, residual tumour volume under the dose-escalated regimen was $1 \%$ as opposed to $11 \%$ under the densified regimen, and in the vascular case residual tumour volume was $6 \%$ as opposed to $35 \%$. In the perfusion-limited scenario, both intensified regimens yielded similar results.

\subsubsection{Average tumour regrowth following Doxorubicin application cycle}

Table 4 presents the tumour's average rate of re-growth per treatment cycle under each simulated regimen. In the diffusion-limited scenario, the average rate of regrowth under the 

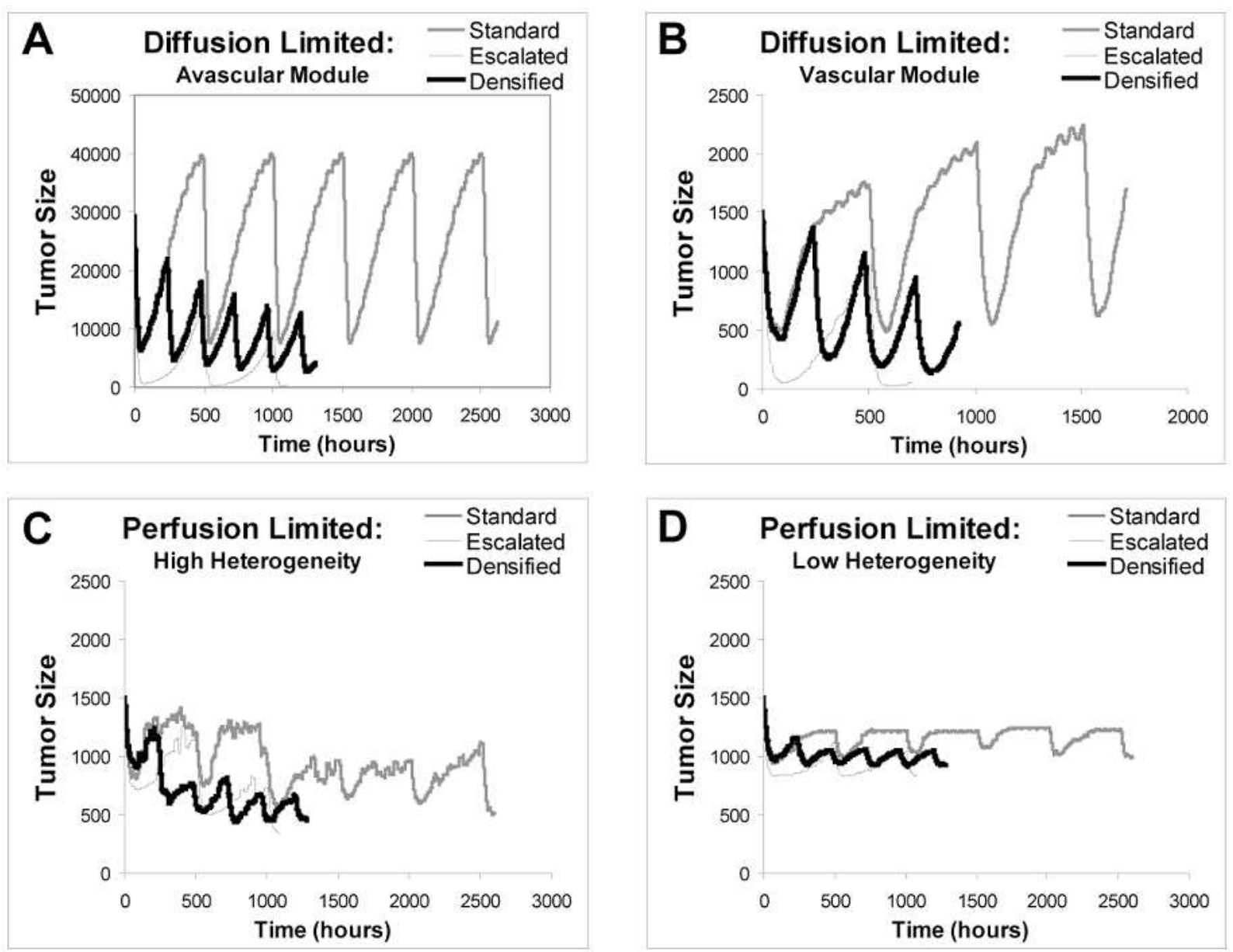

Figure 3: NHL tumour dynamics over time under cyclic standard, dose-escalated or densified Doxorubicin-based regimens (tumour size presented in relative units). Figures 3A, 3B depict the diffusion limited setting (3A: avascular module, 3B: vascular module) and Figures 3C, 3D depict the perfusion-limited setting (3C: high temporal heterogeneity, 3D: low temporal heterogeneity). 
standard regimen exceeded 100\%. Both methods of regimen intensification significantly reduced tumour regrowth between treatment cycles, dose-escalation being the more effective of the two intensification strategies. In the perfusion-limited scenario, the standard and the intensified treatment regimens all produced similar rates of tumour regrowth, though the intensified regimens were slightly more effective than the standard.

\subsubsection{Time below threshold}

We calculated the percentage of time in which the tumour remained below $S_{3 / 4}(3 / 4$ of its initial size), $S_{1 / 2}$ and $S_{1 / 4}$ (see Table 5). In all investigated scenarios, both simulated intensified regimens were significantly more effective than the standard. In the diffusion-limited setting, dose-escalation was the more effective intensification strategy (Table 5C), whereas in the perfusion-limited setting, densification appeared to provide a slight advantage (Table 5B).

\section{Discussion}

We developed a comprehensive mathematical model of residual NHL disease, for addressing the possibility of improving treatment through Doxorubicin-based intensified chemotherapy. Our simulations suggest the existence of a critical Doxorubicin regimen intensity (Critical Intensity: CI). If the regimen intensity is lower than CI, the individual tumours manage to return to their pre-dosing size during the dosing interval.

Consequently, varying regimen intensity in the range below CI will not result in significant improvement, regardless of the dose or the number of treatment cycles. This result could be clearly observed in all simulated cases. The existence of CI, and the inability to maintain the regimen intensity above it, may account for the disappointing results of regimen intensification in some clinical trials [8], as opposed to its success in others [12]. These results further elaborate on the dose intensity paradigm, which has been put forward by Norton [37]: It does not suffice to simply increase the regimen intensity; it must be kept above a certain critical value. Below CI, additional chemotherapy cycles will not be beneficial for the elimination of residual microscopic disease, even in the ideal scenario of a $100 \%$ drug-sensitive tumour cell population. This critical value is determined by specific biological parameters, which can differ between tumour types and chemotherapeutic drugs. Reductions of recommended chemotherapy doses in elderly and other high risk patients, as well as delays of administration of successive chemotherapy cycles due to low blood counts are very frequent in clinical practice. Our results suggest that for each particular pair of a malignant disease and chemotherapy protocol the lower limit of dose intensity which still has significant efficacy (the CI threshold) should be determined in well designed clinical trials. This can avoid administration of potentially toxic treatment that a priori is bound to fail.

While maintaining regimen intensity constant and above CI, we compared the relative efficacy of two treatment intensification strategies: dose-escalation, in which the standard 
inter-dose interval was maintained and the dose administered per cycle was increased, versus regimen densification, in which the standard dose was maintained while the inter-dose interval was reduced. We took tumour heterogeneity into account, examining various diffusion-limited and perfusion-limited scenarios.

In all investigated scenarios, all simulated regimens intensified above CI were significantly more effective than the standard in controlling the residual tumour volume, as well as in maintaining tumour size below a certain threshold. In the diffusion-limited case, the intensified regimens also managed to substantially reduce tumour regrowth between dosing cycles. When comparing the two different intensification strategies, we observed that in the diffusion-limited setting the dose-escalated regimen seemed to have an advantage over the densified regimen. However, simulations performed in the perfusion-limited setting showed both intensified regimens to be similarly effective. Additionally, we note that if regimen intensity is kept above CI, the general behaviour of diffusion-limited tumours is characterized by sequential regressions, meaning that tumour regrowth is reduced after each cycle of chemotherapy. In perfusion-limited cases, however, after a pronounced initial regression during the first few treatment cycles, tumour size remains at a certain plateau despite further treatment (see Figure 3). This plateau may result from the presence of poorly perfused quiescent tumour cell populations, which cannot be eliminated, due to the low drug concentration in their proximity and the insusceptibility of quiescent cells to the drug.

To summarize, both strategies for increasing chemotherapy intensity, namely dose escalation and regimen densification appear to improve efficacy significantly. Therefore, the choice between them in clinic should be based on toxicity considerations. Theoretical work has emphasized the importance of the frequency of drug administration $[1,3,19]$ and it is well known that the primary limitation of dose intensification of cytotoxic chemotherapy is myelotoxicity [28]. Vainstein et al. have implemented a recently developed mathematical model of granulopoiesis to analyze the myelotoxicity caused by different Doxorubicin treatment intensification strategies with and without supportive treatment with Granulocyte Colony-stimulating Factor (G-CSF) [46]. Preliminary results indicate that densification is less toxic than dose-escalation.

Clearly, our results are qualitative and theoretical, and should be interpreted as such. However, the fact that the exploration of three structurally very different tumor growth modules has led to similar conclusions is promising. Further clinical validation of our model is warranted. Further, we suggest that the quantitatively applicable conclusions can be obtained through implementation of our models on a larger scale, with accurate parameters of both tumour growth and intra-tumoural chemotherapy PK/PD.

\section{Appendix: Model Equations}

This appendix specifies some of the equations used in the mathematical model. 


\subsection{Avascular tumour growth module}

\subsubsection{Nutrient and drug diffusion}

The following equations applied in the avascular module are respectively the standard diffusion equation (Equation 1), the diffusion equation after being modified to take into account viable cells' consumption of nutrient or drug ((Equation 2), and their respective boundary conditions ((Equation 3,4). In describing the consumption, we made a slight distinction between nutrient and drug: viable cells are assumed to consume nutrient at a constant rate, whereas drug consumption, which is described by modelling the binding of the drug to target sites in the viable cells, is non-constant and depends on local drug concentration. $C(r, t)$ is the nutrient (or drug) concentration at position $(r, t), K$ is a diffusion coefficient, $P$ is the permeability of the interface and $C_{\text {out }}$ the outer nutrient concentration which is assumed to be constant, or the drug concentration which evolves according to drug pharmacokinetics.

\section{Equation 1}

$$
\frac{\partial C}{\partial t}=\frac{K}{r^{2}} \frac{\partial}{\partial r}\left(r^{2} \frac{\partial C}{\partial r}\right)
$$

\section{Equation 2}

$$
\frac{\partial C}{\partial t}=\frac{K}{r^{2}} \frac{\partial}{\partial r}\left(r^{2} \frac{\partial C}{\partial r}\right)-Q(P, C)
$$

\section{Equation 3}

$$
\left.\frac{\partial C}{\partial t}\right|_{\text {interface }}=P\left(C_{\text {out }}-C\right)
$$

\section{Equation 4}

$$
\left.\frac{\partial C}{\partial t}\right|_{r=0}=0, \quad \forall t
$$

\subsection{Vascular tumour growth module}

\subsubsection{Blood flow}

The flow in each vessel, assuming laminar steady Poiseuille flow, where $\Delta P$ is the pressure drop between two points of the network, $\dot{Q}$ the flow rate in each vessel, $Z, L, r$, and $H$ are respectively the resistance, length, radius, and hematocrit, $\mu$ is the radius and hematocrit dependent viscosity (Alarcon et al. 2004):

$$
\Delta P=\dot{Q} Z
$$




$$
Z=\frac{8 \mu(r, H) L}{\pi r^{4}}
$$

\subsubsection{Vessel structural modification}

Immature vessels' radii are modified at each time step of the simulation according to the following process, where $r_{i m}$ stands for the immature vessel radius, $r_{m a t}$ is the initial radius of the mature vessels, and $\epsilon$ is a random number uniformly distributed in the interval $(0,3)$ according to Willemse et al. (Willemse et al. 1997):

$$
r_{i n}=r_{m a t}(1+\epsilon)
$$

\subsubsection{Nutrient and drug diffusion}

The diffusion equation in the vascular module assuming adiabatic conditions ${ }^{5}$, where $C$ is the concentration of nutrient or drug, $K$ is a diffusion coefficient and $q(x, y)$ the uptake coefficient at position $(x, y)$ :

$$
K \nabla^{2} C-q(x, y) C=0 .
$$

Boundary conditions were prescribed assuming that nutrient and drug molecules enter the system by crossing the walls of the vessels, the flux being given by:

$$
J=-K \nabla C
$$

Mixed boundary conditions imposed on the vessel walls, where $n_{w}$ is the unit vector, orthogonal to the vessel wall, $C_{b}$ is the drug or nutrient concentration in the blood, and $P$ the permeability of the vessel:

$$
-K n_{w} \cdot \nabla C=P\left(C_{b}-C\right) .
$$

No-flux boundary conditions along the edges of the computational domain $\Omega$, where $\left.n\right|_{\partial \Omega}$ is the unit outward vector, orthogonal to the boundary of the domain:

$$
\left.n\right|_{\partial \Omega} \cdot \nabla C=0
$$

\subsubsection{Doxorubicin PK/PD}

The decline of drug concentration in plasma over time is described in the equations below, where $V_{d}$ is the volume of distribution of the drug, $k$ the fraction of drug which is eliminated from the compartment per unit time, inversely related to the half-life $t_{1 / 2}$ :

\footnotetext{
${ }^{5}$ This diffusion equation was solved by means of a two-grid V-cycle multigrid method. Multigrid enables improvement of the rate of convergence of classical numerical methods through interpolation of an initially "rough" solution on a fine grid (Hackbusch 1985; McCormick 1987).
} 


$$
\begin{gathered}
\frac{\partial C_{b}}{\partial t}=-k C_{b}, \\
C_{b}(0)=\frac{d o s e}{V_{d}}, \\
k=\frac{\ln (2)}{t_{1 / 2}} .
\end{gathered}
$$

The survival fraction $S$ (percentage of cells that survives the drug at each time step), where $C_{b}(t)$ is the relevant drug concentration and $a, E c_{1 / 2}$ are constants:

$$
S=\frac{a C_{b}}{C_{b}+E c_{1 / 2}}
$$

Acknowledgements. The work was supported by the Chai Foundation and by the EU Research Training Network (HPRN-CT-2000-00105: "Using mathematical modelling and computer simulations to improve cancer therapy"). The authors wish to thank Professor Dina Ben Yehuda for reviewing the manuscript.

\section{References}

[1] Z. Agur. Randomness, synchrony and population persistence. J. Theor. Biol., 112 (1985), 677-693.

[2] Z. Agur, L. Arakelyan, P. Daugulis, Y. Ginosar. Hopf point analysis for angiogenesis models. Discrete and Continuous Dynamical Systems - Series B, 4 (2004), No. 1, 29-38.

[3] Z. Agur, R. Arnon, B. Schechter. Reduction of cytotoxicity to normal tissues by new regimens of phase-specific drugs. Math. Biosci. 92 (1988), 1-15.

[4] T. Alarcon, H.M. Byrne, P.K. Maini. A Cellular Automaton Model for Tumor Growth in Homogeneous Environment. J. Theor. Biol., 225 (2004), pp. 257-74.

[5] B. Alberts, D. Bray, J. Lewis, M. Raff, K. Roberts, J.D. Watson. Molecular biology of the cell. Garland Publishing, New York and London, 1994.

[6] L. Arakelyan, Y. Merbl, Z. Agur. Vessel maturation effects on tumour growth: validation of a computer model in implanted human ovarian carcinoma spheroids. Eur. J. Cancer, 41 (2005), No 1, 159-167.

[7] L. Arakelyan, V. Vainstein, Z. Agur. A computer algorithm describing the process of vessel formation and maturation and its use for predicting the effects of anti-angiogenic and multi-maturation therapy on vascular tumor growth. Angiogenesis 5 (2002), 203-214. 
[8] M. Balzarotti, M. Spina, B. Sarina, M. Magagnoli, L. Castagna, I. Milan, C. Ripa, F. Latteri, D. Bernardi, A. Bertuzzi, A. Nozza, M. Roncalli, E. Morenghi, U. Tirelli, A. Santoro. Intensified CHOP regimen in aggressive lymphomas: maximal dose intensity and dose density of doxorubicin and cyclophosphamide. Ann. Oncol. 13 (2002), 1341-6.

[9] S.C. Barranco. Cellular and molecular effects of adriamycin on dividing and nondividing cells. Pharmacol. Ther., 24 (1984), 303-19.

[10] R.C. Bast, D.W. Kufe, R.E. Pollock, R.R. Weichselbaum, J.F. Holland. Cancer Medicine. BC Decker Inc, Canada, 2000.

[11] L.E. Benjamin, D. Golijanin, A. Itin, D. Pode, E. Keshet. Selective ablation of immature blood vessels in established human tumors follows vascular endothelial growth factor withdrawal. J. Clin. Invest. 103 (1999), 157-158.

[12] D.W. Blayney, M.L. LeBlanc, T. Grogan, E.R.Gaynor, R.A. Chapman, Spiridonidis C.H., Taylor S.A., Bearman S.I., Miller T.P., R.I. Fisher. Dose-intense chemotherapy every 2 weeks with dose-intense cyclophosphamide, doxorubicin, vincristine, and prednisone may improve survival in intermediate- and high-grade lymphoma: a phase II study of the Southwest Oncology Group (SWOG 9349). J. Clin. Oncol. 21 (2003), No. 13, 2466-73.

[13] C.C. Boring, T.S. Squires, T. Tong, S. Montgomery. Cancer statistics. CA Cancer J. Clin. 44 (1994), 7-26.

[14] P.P. Brons, J.M. Raemaekers, M.J. Bogman, P.E. van Erp, J.B. Boezeman, A.H. Pennings, H.M. Wessels, C. Haanen. Cell cycle kinetics in malignant lymphoma studied with in vivo iodeoxyuridine administration, nuclear Ki-67 staining, and flow cytometry. Blood, 80 (1992), No. 9, 2336-43.

[15] H.M. Byrne, M.A. Chaplain. Growth of nonnecrotic tumors in the presence and absence of inhibitors. Math. Biosci., 134 (1995), No. 2, 151-181.

[16] H.M. Byrne, M.A. Chaplain. Growth of necrotic tumors in the presence and absence of inhibitors. Math. Biosci., 135 (1996), No. 2, 187-216.

[17] J.J. Casciari, S.V. Sotirchos, R.M. Sutherland. Mathematical modelling of microenvironment and growth in EMT6/Ro multicellular tumor spheroids. Cancer Res. 25, (1992), No. 1, 1-22.

[18] M.L. Citron, D.A. Berry, C. Cirrincione, C. Hudis, E.P. Winer, W.J. Gradishar, N.E. Davidson, S. Martino, R. Livingston, J.N. Ingle, E.A. Perez, J. Carpenter, D. Hurd, J.F. Holland, B.L. Smith, C.I. Sartor, E.H. Leung, J. Abrams, R.L. Schilsky, H.B. Muss, L. Norton. Randomized trial of dose-dense versus conventionally scheduled and sequential versus concurrent combination chemotherapy as postoperative adjuvant 
treatment of node-positive primary breast cancer: first report of Intergroup Trial C9741/Cancer and Leukemia Group B Trial 9741. J. Clin. Oncol., 21 (2003), 1431-1439.

[19] L. Cojocaru, Z. Agur. A theoretical analysis of interval drug dosing for cell-cycle-phase-specific drugs. Math. Biosci., 109 (1992), 85-97.

[20] M.S. Czuczman, R. Weaver, B. Alkuzweny, J. Berlfein, A.J. Grillo-Lopez. Prolonged clinical and molecular remission in patients with low-grade or follicular non-Hodgkin's lymphoma treated with rituximab plus CHOP chemotherapy: 9-year follow-up. J. Clin. Oncol., 22 (2004), No. 23, 4711-6.

[21] A. Deutsch, S. Dormann. Modeling of avascular tumor growth with a hybrid cellular automaton. In Silico Biol. 2 (2002), 1-14.

[22] M. Erlanson, J. Lindth, B. Zackrisson, G. Landberg, G. Roos. Cell kinetic analysis of non-Hodgkin's lymphomas using in vivo iodeoxyuridine incorporation and flow cytometry. Hematol. Oncol., 13 (1995), 207-17.

[23] B. Fisher, S. Anderson, A. DeCillis, N. Dimitrov, J.N. Atkins, L. Fehrenbacher, P.H. Henry, E.H. Romond, K.S. Lanier, E. Davila, C.G. Kardinal, L. Laufman, H.I. Pierce, N. Abramson, A.M. Keller, J.T. Hamm, D.L. Wickerham, M. Begovic, E. Tan-Chiu, W. Tian, N. Wolmark. Further evaluation of intensified and increased total dose of cyclophosphamide for the treatment of primary breast cancer: findings from National Surgical Adjuvant Breast and Bowel Project B-25. J. Clin. Oncol., 17 (1999), 3374-88.

[24] W. Hackbusch. Multigrid methods and applications. Springer-Verlag, Berlin, 1985.

[25] D. Hasenclever, M. Loeffler, V. Diehl. Rationale for dose escalation of first line conventional chemotherapy in advanced Hodgkin's disease. Ann. Oncol., 7 (1996), Suppl. 4, S95-S98.

[26] I.C. Henderson, D.A. Berry, G.D. Demetri, C.T. Cirrincione, L.J. Goldstein, S. Martino, J.N. Ingle, M.R. Cooper, D.F. Hayes, K.H. Tkaczuk, G. Fleming, J.F. Holland, D.B. Duggan, J.T. Carpenter, E. Frei, R.L. Schilsky, W.C. Wood, H.B. Muss, L. Norton. Improved outcomes from adding sequential Paclitaxel but not from escalating Doxorubicin dose in an adjuvant chemotherapy regimen for patients with node-positive primary breast cancer. J. Clin. Oncol. 21 (2003), 976-83.

[27] W.M. Hryniuk. Average relative dose intensity and the impact on design of clinical trials. Semin. Oncol. 14 (1987), 65-74

[28] K. Itoh, T. Ohtsu, H. Fukuda, Y. Sasaki, M. Ogura, Y. Morishima, T. Chou, K. Aikawa, N. Uike, F. Mizorogi, T. Ohno, S. Ikeda, T. Sai, M. Taniwaki, F. Kawano, M. Niimi, T. Hotta, M. Shimoyama, K. Tobinai. Randomized phase II study of biweekly 
CHOP and dose-escalated CHOP with prophylactic use of lenograstim (glycosylated G-CSF) in aggressive non-Hodgkin's lymphoma: Japan Clinical Oncology Group Study 9505. Ann. Oncol. 13 (2002), 1347-1355.

[29] R.K. Jain. Delivery of molecular and cellular medicine to solid tumors. Adv. Drug. Deliv. Rev. 46 (2001), 146-68.

[30] L. Jana, Gevertza, S. Torquato. Modeling the effects of vasculature evolution on early brain tumor growth. Journal of Theoretical Biology, 243 (2006), 517.

[31] L.A. Knuz-Schughart, M. Kreutz, R. Knuechel. Multicellular spheroids: a three-dimensional in vitro culture system to study tumor biology. Int. J. Exp. Pathol. 79 (1988), 1-23.

[32] M.A. Konerding, E. Fait, A. Gaumann. 3D microvascular architecture of pre-cancerous lesions and invasive carcinomas of the colon. Br. J. Cancer., 84 (2001), 1354-1362.

[33] Y. Li, H. Nagai, T. Ohno, M. Yuge, S. Hatano, E. Ito, N. Mori, H. Saito, T. Kinoshita. Aberrant DNA methylation of p57(KIP2) gene in the promoter region in lymphoid malignancies of B-cell phenotype. Blood, 100 (2002), No. 7, 2572-7.

[34] L.A. Liotta, G.M. Saidel, J. Kleinerman. Diffusion model of tumor vascularization and growth. Bull. Math. Biol., 39 (1977), 117-128.

[35] M. Marusi, Z. Bajzer, J.P. Freyer, S. Vuk-Pavlovi. Analysis of growth of multicellular tumor spheroids by mathematical models. Cell Prolif., 27 (1994), 73-94.

[36] S.F. McCormick. Multigrid methods, SIAM, Philadelphia, PA, 1987.

[37] L. Norton. Theoretical concepts and the emerging role of taxanes in adjuvant therapy. Oncologist, 6 (2000), Suppl. 3, 30-5.

[38] M.E. Orme, M.A. Chaplain. A mathematical model of vascular tumor growth and invasion. Math. Comp. Modelling, 23 (1996), 43-60.

[39] A.A. Patel, E.T. Gawlinski, S.K. Lemieux, R.A. Gatenby. A cellular automaton model of early tumor growth and invasion. J. Theor. Biol., 213 (2001), No. 3, 315-31.

[40] M. Pfreundschuh, L. Trumper, M. Kloess, R. Schmits, A.C. Feller, C. Rube, C. Rudolph, M. Reiser, D.K. Hossfeld, H. Eimermacher, D. Hasenclever, N. Schmitz, M. Loeffler. German High-Grade Non-Hodgkin's Lymphoma Study Group. Two-weekly or 3-weekly CHOP chemotherapy with or without etoposide for the treatment of elderly patients with aggressive lymphomas: results of the NHL-B2 trial of the DSHNHL. Blood, 104 (2004), No. 3, 634-41 
[41] A.R. Pries, T.W. Secomb, P. Gaehtgens. Structural adaptation and stability of microvascular networks: theory and simulations. Am. J. Physiol., 275 (1998), H349-H360.

[42] A.R. Pries, T.W. Secomb, T. Gessner, M.B. Sperandio, J.F. Gross, P. Gaehtgens. Resistance to blood flow in microvessels in vivo. Circ. Res., 75 (1994), 904-915.

[43] B. Ribba, T. Alarcon, K. Marron, P.K. Maini, Z. Agur. Doxorubicin treatment efficacy on non-Hodgkin's lymphoma: Computer model and simulations. Bull. Math. Biol., 67 (2004), No. 1, 79-99.

[44] T. Stokke, H. Holte, L. Smedshammer, E.B. Smeland, O. Kaalhus, H.B. Steen, 1998, "Proliferation and apoptosis in malignant and normal cells in B-cell non-Hodgkin's lymphomas," Br. J. Cancer. 77(11), pp. 1832-8.

[45] G. Todeschini, S. Gelio, C. Tecchio. Rituximab-CHOP or two-weekly $C H O P+G$-CSF in aggressive lymphoma of the elderly. Ann. Oncol., 15 (2004), No. 3, 538-539.

[46] V. Vainstein, Y. Ginosar, M. Shoham, D.O. Ranmar, A. Ianovski, Z. Agur. The complex effect of granulocyte colony-stimulating factor on human granulopoiesis analysed by a new physiologically-based mathematical model. J. Theor. Biol., 234 (2005), No. 3, 311-327.

[47] V. Vainstein, Y. Ginosar, M. Shoham, A. Ianovski, A. Rabinovich, Y. Kogan, V. Selitser, Z. Agur. Improving cancer therapy by doxorubicin and granulocyte colony-stimulating factor: insights from a computerized model of human granulopoiesis. Math. Model. Nat. Phen., 1 (2006), No. 2.

[48] F. Willemse, M. Nap, H.W.A. de Bruijn, H. Hollema. Quantification of vascular density and of lumen and vessel morphology in endometrial carcinoma. Evaluation of their relation to serum levels of tissue polypeptide-specific antigen and CA-125. Anal. Quant. Cytol. Histol., 1 (1997), 1-7.

[49] G.D.Yancopoulos, S. Davis, N.W. Gale, J.S. Rudge, S.J. Wiegand, J. Holash. Vascular specific growth factors and blood vessel formation. Nature, 407 (2000), 242-248. 
Table 1: Model Parameters

\begin{tabular}{|c|c|c|c|c|}
\hline $\begin{array}{l}\text { Parameter } \\
r_{m a t}\end{array}$ & $\begin{array}{l}\text { Description } \\
\text { Mature vessel radius }\end{array}$ & $\begin{array}{l}\text { value } \\
20\end{array}$ & $\begin{array}{l}\text { Unit } \\
\mu m\end{array}$ & $\begin{array}{l}\text { Ref. } \\
\text { Jain, } 2001\end{array}$ \\
\hline$r_{i m}$ & Immature vessel radius & $20<r_{i m}<60$ & $\mu m$ & Jain 2001 \\
\hline$K_{\text {glucose }}$ & Glucose diffusion coefficient & $2.3 \cdot 10^{-7}$ & $\mathrm{~cm}^{2} \cdot \mathrm{s}^{-1}$ & Nagai et al. 2002 \\
\hline$q$ & Glucose uptake coefficient & $13.2 \cdot 10^{-11}$ & $m M \cdot m^{-1}$ & Norton 2000 \\
\hline$K_{\text {doxorubian }}$ & Doxorubicin diffusion coefficient & $2.7 \cdot 10^{-10}$ & $c m^{2}: s^{-1}$ & Orme \& Chap lain 1996 \\
\hline$C_{b}$ & Glucose concentration in blood & 5 & $m M$ & Patel et al. 2001 \\
\hline$P$ & Vessel permeability & $3.0 \cdot 10^{-4}$ & $\mathrm{~cm} \cdot \mathrm{s}^{-l}$ & Pries et al. 1998 \\
\hline$V_{d}$ & Doxo. volume of distribution & 682 & $L: m^{2}$ & Pries et al. 1994 \\
\hline$t_{t / 2}$ & Doxo. half life & 26 & $h$ & Pries et al. 1994 \\
\hline$a$ & Doxo. PD coefficient & 0.095 & & Ribba et al. 2005 \\
\hline$E c_{1 / 2}$ & Doxo. effective concentration & 0.39 & $m g \cdot L^{-I}$ & Stokke et al. 1998 \\
\hline
\end{tabular}

Table 2: Cell cycle kinetic parameters for High Grade NHL (Cuczman et al. 200-)

\begin{tabular}{|l|ll|}
\hline Parameter & Value & Range \\
\hline S-INA (\%) & 25.2 & $(3.6-37.1)$ \\
GF (\%) & 34 & $(15-93)$ \\
\hline$T_{s}(h)$ & 19.1 & $(9.7-33.6)$ \\
\hline$T_{c}(h)$ & 57 & $(21-93)$ \\
$T_{G 1}(h)$ & 36 & $(11-59)$ \\
\hline
\end{tabular}


Table 3: Residual Tumour Vohme

\begin{tabular}{|lll|l|}
\hline & Standard & Dose-escalated & Densified \\
\hline Diffusion-limited Avascular module & $32 \%$ & $1 \%$ & $11 \%$ \\
Diffusion-limited Vascular module & $135 \%$ & $6 \%$ & $35 \%$ \\
Perfusion-limited High heterogeneity & $43 \%$ & $37 \%$ & $34 \%$ \\
Perfusion-limited Low heterogeneity & $74 \%$ & $60 \%$ & $66 \%$ \\
\hline
\end{tabular}

Residual tumour volume (size of tumour at end of simulation / size of tumour at beginning) in all modelled pathophysiological scenarios, under periodic standard, dose-escalated and densified Doxorubicin treatment regimens.

Table 4: Average tumour regrowth between cycles

\begin{tabular}{|l|l|lc|}
\hline & Standard & Dose-escalated & Densified \\
Diffusion-limited Avascular module & $105 \%$ & $57 \%$ & $83 \%$ \\
\hline Diffusion-limited Vascular module & $119 \%$ & $79 \%$ & $85 \%$ \\
\hline Perfusion-limited High heterogeneity & $90 \%$ & $87 \%$ & $88 \%$ \\
\hline Perfusion-limited Low heterogeneity & $97 \%$ & $95 \%$ & $95 \%$ \\
\hline
\end{tabular}

Average tumour regrowth between drug administration cycles (size of tumour at end of dosing cycle/size of tumour at beginning of dosing cycle) in all modelled pathophysiological scenarios, under periodic standard, dose-escalated and densified Doxorubicin treatment regimens. 
Table 5: Time Below $S_{3 / 4} S_{1 / 2}$ and $S_{1 / 4}$ Thresholds

\begin{tabular}{|c|c|c|c|c|}
\hline $\begin{array}{l}\text { Threshold } \\
\text { value }\end{array}$ & Module & Standard & $\begin{array}{l}\text { Irose- } \\
\text { escalated }\end{array}$ & Densified \\
\hline \multirow[t]{3}{*}{ A. $S_{3 / 4}$} & Diffusion-limited/Av ascular module & $45 \%$ & $100 \%$ & $100 \%$ \\
\hline & Diffusion-limited/Vascular module & $30 \%$ & $91 \%$ & $92 \%$ \\
\hline & $\begin{array}{l}\text { Perfusion-limited/High heterogeneity } \\
\text { Perfusion-limited/Low heterogeneity }\end{array}$ & $\begin{array}{l}85 \% \\
42 \%\end{array}$ & $97 \%$ & $\begin{array}{l}98 \% \\
90 \%\end{array}$ \\
\hline \multirow[t]{3}{*}{ B. $S_{1 / 2}$} & Diffusion-limited/Av ascular module & $27 \%$ & $98 \%$ & $89 \%$ \\
\hline & Diffusion-limited/Vascular module & $15 \%$ & $75 \%$ & $74 \%$ \\
\hline & $\begin{array}{l}\text { Perfusion-limited/High heterogeneity } \\
\text { Perfusion-limited/Low heterogeneity }\end{array}$ & $\begin{array}{l}25 \% \\
0 \%\end{array}$ & $\begin{array}{l}35 \% \\
0 \%\end{array}$ & $\begin{array}{l}65 \% \\
0 \%\end{array}$ \\
\hline \multirow[t]{3}{*}{ C. $S_{1 / 4}$} & Diffusion-limited/Av ascular module & $3 \%$ & $84 \%$ & $46 \%$ \\
\hline & Diffusion-limited/Vascular module & $0 \%$ & $57 \%$ & $36 \%$ \\
\hline & $\begin{array}{l}\text { Perfusion-limited/High heterogeneity } \\
\text { Perfusion-limited/Low heterogeneity }\end{array}$ & $\begin{array}{l}0 \% \\
0 \%\end{array}$ & $\begin{array}{l}0 \% \\
0 \%\end{array}$ & $\begin{array}{l}1 \% \\
0 \%\end{array}$ \\
\hline
\end{tabular}

Percentage of time tumour is maintained below the threshold value of $3 / 4(A), 1 / 2(B)$ and $1 / 4(C)$ of its original size in all modelled pathophysiological scenarios, under periodic standard, dose-escalated and densified Doxorubicin treatment regimens. 